\title{
Multitherapy treating autism (autistic spectrum disorder) and down syndrome.
}

\author{
Anna Lou Olivier* \\ CREM (Centro de Referência e Estudo em Multiterapia), São Paulo, SP, Brazil
}

\begin{abstract}
This study did not bother to detect causes, brain impairment or degree of autism or Down syndrome. Therefore, no tests or exams were applied. Nor have other recent studies have been compared because the intention was not to make comparisons but to seek a new form of treatment. The target children of this study were already diagnosed of their respective clinical appointments and were immediately started on treatment. The main objective was to achieve greater control of symptoms and seizures, using the Multi-Therapy Method. This method, despite using some existing techniques, is innovative and stands out for adapting to each patient and shaping a single treatment that can be extended to a group, provided that this group has identical characteristics. The purpose of this study was to prove efficient of Multitherapy in treatment of Autism and Down syndrome. All the results were practical, not measured by examinations or tests, but by results in real life. In two periods concluded, was attending 83 children, 60 with autism and 23 with Down syndrome. All children Down syndrome made good progress, especially in learning. Of the 60 children attended, 50 improved the communication with the external environment, being this the main objective of this treatment. The children developed interaction with the external environment and could be socialized, according to their personal characteristics. The other 10 children had severe autism and did not have as satisfactory results as the others, but they had a slight improvement, especially in the control of the collapses, in a situation of stress. Therefore, it is considered that, even in the most severe cases of autism, there has been an improvement. In percentage, $100 \%$ Down syndrome and 83\% Autism made good progress.
\end{abstract}

Keywords: Multitherapy treatment, New method of treatment, Autism, Autistic spectrum disorder.

Accepted on January 29, 2018

\section{Objective}

To prove that Multitherapy is more effective than the current methods used for the treatment of Autism (Autism Spectrum Disorder).

\section{Introduction}

To understand how the Autistic brain works, it is necessary first to know how the normal brain functions. I will not delve into this; I will only try to give a sense of how this occurs. Stimuli reach the brain through all sensory pathways: sight, hearing, smell, taste, and touch (five senses). And it is now considered an area of equilibrium as a possible sixth sense, far from being the esoteric sixth sense; it is located in a part of the brain next to the channel that leads the sound to recognition of the brain, so to speak. The inner ear / labyrinth is formed by two systems, the cochlear (sensory organ responsible for the decoding of the Sons) and the vestibular (they are semicircular canals sacculus and utricle that inform the Central Nervous System about the position and movements of the head). The sound: Sound stimulus, which is actually a vibration, is picked up and encoded in the cochlea and transmitted to the brain by the auditory nerve. In the brain, groups of specialized neurons (nuclei) decode the vibration taking it as a message driven until it reaches the auditory cortex. When arriving the message is as faithful as possible of the original stimulus, but it must be understood that it has crossed a path to reach the point of understanding. The sound is picked up by the external ear, amplified by the middle ear and transmitted to the inner ear where the cochlea transforms the sound vibration into a nervous message. The vision: Retina, located at the back of the eye, encodes the light (luminous stimuli). The visual messages pass through the optic nerve and blind the brain in a very detailed way (intensity of light, colors, sizes, etc.). This information is processed and ends in the visual area of the occipital cortex where perception occurs. The bottom line is that in the brain, different centers analyze stimuli and messages and decode them by producing perception at the cortex level. In autism this does not occur or, if it occurs, is disordered or unconventional.

\section{Method}

The Multi-therapy has techniques that supplement this deficiency in the reception of the stimuli. In many cases, it is able to penetrate into the world of the autistic, arousing sensitivity, creativity and greater perception of the external world. Multitherapy seeks to treat not only Autism, but Hyperlexia which, in some cases, is characteristic of Autism and also treat cases in which mental retardation impedes learning $[1,2]$. Lighter cases tend to have very fast responses. Very serious cases may not have a rapid 
response, but although in the long-term, they show improvement. Multitherapy uses music therapy in a continuous and original way with specific sounds and rhythms that fit each brain and stimulates learning and awakening. Art Therapy is also used in a thorough and complete way (biodance, psychodrama, theatrical techniques, drawing), as well as different forms of breathing, relaxation, stimuli of memory and sensations, among other resources. Multitherapy is a combination of therapies aimed at the best treatment of disorders and dysfunctions because it is artisan, analyzing and treating each case as unique and isolated, it is one of the best options in the treatment of any disorder, especially Autism that needs special attention. So the first strong point is that each patient has a treatment developed only for their case. Considering that each organism has its own characteristics and reacts in a certain way to the stimuli and treatments, the method must be shaped in each case. In general, the various techniques adapted together in each case, make the treatment faster, more efficient and practical. For each case the best techniques and areas to be merged are stipulated, all according to the characteristics of the Multitherapy. It is holistic, in the original sense of treating the individual completely and not only in one aspect (psychological, neurological, physical, etc.) This study began in 1985 and 10 autistic children and 20 children with Down's Syndrome were treated using the Multi-Therapy technique developed by Olivier [3,4]. The following year, the number of children with Down syndrome decreased to 3 , and the majority of autistic children (20) in these studies were discontinued after one year. In 2003 the studies were restarted, in a private clinic Psiconeuroarte and special schools, attending 30 autistic children. From 2005 the results began to be published in textbooks and articles in specialized magazines. Method Multitherapy was registered as patrimony of CREM (Centro de Referência e Estudo em Multitherapia) Brazil [5,6]. Better innovation in this study is all the results were practical, not measured by examinations or tests, but by results in real life. Children were performing in practice the better of themselves without preoccupation with rules or standardized tests. On 10/11/2014, Multitherapy as an ally in the social and behavioral education of the autistic was the theme defended by the psychotherapist Olivier during the public hearing Citizenship of the Autistic, within the $2^{\text {nd }}$ Seminar of Space Women Studies, held in the Legislative Assembly of the State of São Paulo - Brazil. From this date on, the technique of Multitherapy for the treatment of autistic patients in Brazil became official $[7,8]$.

\section{Results}

Very serious cases are mitigated more quickly and efficiently than the treatments used today; Lighter cases are solved, usually with very positive results, especially in the stimulation of speech, hearing and in the development of artistic creativity. Unique insight of this study is unique insight by Anna Lou Olivier. Treatment of Autism (or other disturbances) needs new method more practice without rules or standardized tests. Aiming each case as unique and bringing control or healing and not just numbers in the searches of researches.

\section{Discussion and Conclusion}

Multitherapy does not intend or advise interruption or denial of medication use. Even in very serious cases requiring medication, multitherapy can and should be associated with neurological/ psychiatric treatment. The main intention is to bring a new form of treatment that can be effective in as autism far as Down syndrome that was also the object of study. A total of 60 autistic children and 23 with Down syndrome were analyzed. Due to the excellent results with Down's syndrome, it was preferred to continue with autistic children only since they had more symptoms, less tolerance and more need for follow-up. In numbers, it was concluded that, of the 60 children attended, 50 improved the communication with the external environment, being this the main objective of this treatment. The children developed interaction with the external environment and could be socialized, according to their personal characteristics. The other 10 children had severe autism and did not have as satisfactory results as the others, but they had a slight improvement, especially in the control of the collapses, in a situation of stress. Therefore, it is considered that, even in the most severe cases of autism, there has been an improvement. As a complement to this research, the 23 children with Down syndrome made good progress, especially in learning. This enhances the effectiveness of the method.

\section{References}

1. Olivier LD. Multitherapy, welcome multitherapy, welcome to the therapy of future. J Psychol Psychother. 2016;6:5.

2. Olivier LD. Multitherapy - Therapy techniques developed by Lou de Olivier. Ment Health Addict Res. 2016;1.

3. Olivier LD. Multitherapy as allied in the social and behavioral education of the autistic. Citizenship of the Autistic. Legislative Assembly of the State of São Paulo Brazil. 2014.

4. Olivier LD. Behavior disorders and learning disabilities describing the multitherapy. Psyche 81 Dossier magazine. 2012;6.

5. Olivier LD: Learning and behavior disorders. Rio de Janeiro - RJ - Brazil. 6 $6^{\text {th }}$ Edn. 2013.

6. Olivier LD. Learning/behavior disorders - Truths that no one published. São Paulo - SP - Brazil. 2003.

7. Olivier LD. Learning problems in preschool. São Paulo - SP - Brazil. 1999.

8. Olivier LD. The productive school. São Paulo - SP - Brazil. 1999.

\section{*Correspondence to:}

\section{Anna Lou Olivier}

CREM (Centro de Referência e Estudo em

Multiterapia)

São Paulo - SP

Brazil

Tel: 05511-973743680

E-mail: louevoce@loudeolivier.com 